\title{
Optimal Length and Signal Amplification in Weakly Activated Signal Transduction Cascades
}

\author{
Madalena Chaves, ${ }^{*}+, *$ Eduardo D. Sontag, ${ }^{\dagger}$ and Robert J. Dinerstein ${ }^{*}$ \\ Department of Mathematics, Rutgers University, New Brunswick, New Jersey 08903, and \\ Lead Generation Informatics, Aventis, Bridgewater, New Jersey 08807 \\ Received: March 9, 2004; In Final Form: July 13, 2004
}

\begin{abstract}
Weakly activated signaling cascades can be modeled as linear systems. The input-to-output transfer function and the internal gain of a linear system provide natural measures for the propagation of the input signal down the cascade and for the characterization of the final outcome. The most efficient design of a cascade for generating sharp signals is obtained by choosing all the off rates equal and a "universal" finite optimal length.
\end{abstract}

\section{Introduction}

Protein kinase cascades are major functional modules used by cells to translate signals generated by receptor activation into diverse biochemical and physiological responses. ${ }^{1}$ Highly conserved throughout evolution and across species, the kinase cascade motif participates in the control of many processes, including cell cycle regulation, gene expression, cellular metabolism, stress responses, and $\mathrm{T}$ cell activation. For this reason, control of kinase cascades by therapeutic intervention has become an attractive area for drug discovery, particularly in the areas of cancer and inflammation. ${ }^{2,3}$

Some four mitogen-activated protein kinase (MAPK) signaling cascades have been found in yeast, ${ }^{4}$ and at least a dozen MAPK cascades have been identified in mammalian cells. ${ }^{5}$ The intensive study of MAPK pathways has prompted efforts to characterize these systems theoretically (see, inter alia, refs 6-14). In this paper, we will utilize concepts and methods from the theory of linear control systems to characterize kinase signaling cascades, in particular the MAPK pathway, to understand how the number of kinases in a cascade and their individual enzymatic activities can affect the pathway in its role as a signal-transducing module. For recent applications of control theory methods to biological systems, see refs 15 and 16 .

Let $R$ denote the input signal, $\tilde{X}_{i}$, the inactive (nonphosphorylated) form of kinase $i$ and $X_{i}$, the active (phosphorylated) form of kinase $i$. The rate constant (or "on" rate) for the $i$ th kinase phosphorylation will be denoted by $\tilde{\alpha}_{i}$, and the dephosphorylation rate constant (or "off" rate) will be denoted by $\beta_{i}$. The input signal $R$ might represent, for example, the concentration of activated receptors, and the dynamics of the signal transduction pathway may be modeled as follows (see ref 10)

$$
\frac{\mathrm{d} X_{1}}{\mathrm{~d} t}=\tilde{\alpha}_{1} R \tilde{X}_{1}-\beta_{1} X_{1} \quad \frac{\mathrm{d} X_{i}}{\mathrm{~d} t}=\tilde{\alpha}_{\mathrm{i}} X_{i-1} \tilde{X}_{i}-\beta_{i} X_{i} \quad i=2, \ldots, n
$$

Assuming that the total amount of kinase $i$ remains constant, that is, $\tilde{X}_{i}+X_{i}=X_{\text {tot }, i}$, the differential equations (1) can be rewritten as

* To whom correspondence may be addressed. E-mail: madalena@ math.rutgers.edu.

Rutgers University.

$\doteqdot$ Aventis.

$$
\frac{\mathrm{d} X_{1}}{\mathrm{~d} t}=\alpha_{1} R\left(1-\frac{X_{1}}{X_{\mathrm{tot}, 1}}\right)-\beta_{1} X_{1}
$$

and

$$
\frac{\mathrm{d} X_{i}}{\mathrm{~d} t}=\alpha_{i} X_{i-1}\left(1-\frac{X_{i}}{X_{\mathrm{tot}, i}}\right)-\beta_{i} X_{i} \quad i=2, \ldots, n
$$

where $\alpha_{i}=\tilde{\alpha}_{i} X_{\text {tot }, i}$. Throughout this paper, we will focus on the case of weakly activated pathways, by which we mean a low level of kinase phosphorylation, that is

$$
X_{i} \ll X_{\mathrm{tot}, i} \Longrightarrow 1-\frac{X_{i}}{X_{\mathrm{tot}, i}} \approx 1
$$

In this case, the equations (2) are simplified to a linear system of the form

$$
\frac{\mathrm{d} X_{1}}{\mathrm{~d} t}=\alpha_{1} R-\beta_{1} X_{1} \quad \frac{\mathrm{d} X_{i}}{\mathrm{~d} t}=\alpha_{i} X_{i-1}-\beta_{i} X_{i} \quad i=2, \ldots, n
$$

In section 2, we will describe how to compute the transfer function and internal gain for this system, and then in section 3 , we will define a set of measures for the output signal, which closely follow those discussed in ref 10 . In section 4 , we prove that the most efficient cascade design for generating sharp signals has equal on rates and a finite length depending only on the cascade's internal gain. In section 5, positive feedback from the last activated kinase to the first is added to the cascade, and the optimal design is re-examined in this new context. Finally, in section 6, we briefly discuss how to check the cascade's stability to random small perturbations.

\section{Input-to-Output Transfer Function}

We will consider the signaling cascade (4) as a system with an input $R$, and an output which will be some function of the concentration of the last kinase $X_{n}$. A typical function consists of the total accumulated concentration of $X_{n}$ or $\int{ }^{t} X_{n}\left(t^{\prime}\right) \mathrm{d} t^{\prime}$. From the biological point of view, however, one should also consider the degradation rate of $X_{n}$ as well as the possibility that some fraction of $X_{n}$ is not active. We will assume that the loss of accumulated kinase (due to degradation or inactivation, for instance) is reflected by a constant rate $l$, and we introduce the output as a new variable, $X_{n+1}$, given by 


$$
\frac{\mathrm{d} X_{n+1}}{\mathrm{~d} t}=X_{n}-l X_{n+1}
$$

(From the control theory point of view, this corresponds to extending the cascade one more step to include a "leaky" integrator.) The variable $X_{n+1}$ expresses the effective concentration of the last kinase. Note that the case $l=0$ recovers the total accumulated kinase, i.e., $X_{n+1}=\int{ }^{t} X_{n}\left(t^{\prime}\right) \mathrm{d} t^{\prime}$.The model for a weakly activated signal transduction cascade may then be written in the more compact form

$$
\frac{\mathrm{d} X}{\mathrm{~d} t}(t)=A X(t)+B R(t) \quad Y(t)=C X(t)
$$

where $X=\left(X_{1}, X_{2}, \ldots, X_{n}, X_{n+1}\right)^{\prime}$ is a column vector whose elements are always nonzero, and $A \in \mathbb{R}^{(n+1) \times(n+1)}, B \in \mathbb{R}^{(n+1) \times 1}$, and $C \in \mathbb{R}^{1 \times(n+1)}$ are the matrices

$$
A=\left(\begin{array}{lllllll}
-\beta_{1} & 0 & 0 & \ldots & 0 & 0 & 0 \\
\alpha_{2} & -\beta_{2} & 0 & \ldots & 0 & 0 & 0 \\
0 & \alpha_{3} & -\beta_{3} & \ldots & 0 & 0 & 0 \\
0 & 0 & \alpha_{4} & \ldots & 0 & 0 & 0 \\
\vdots & & & \ddots & & & \vdots \\
0 & 0 & 0 & \ldots & \alpha_{n} & -\beta_{n} & 0 \\
0 & 0 & 0 & \ldots & 0 & 1 & -l
\end{array}\right) \mathrm{B}=\left(\begin{array}{llll}
0 \\
0
\end{array}\right.
$$

It is well known (see refs 17 and 18, or any other book on control systems) that, for a system such as 5 , the output can be computed directly as the convolution between the input signal $R$ and the impulse response of the system. The impulse response of the system is the output corresponding to a single input pulse. If we let $G$ denote the impulse response (and assuming that the system starts at rest, with initial condition $X(0)=0)$, then

$$
Y(t)=\left(G^{*} R\right)(t)
$$

The impulse response, $G$, characterizes the action that the internal structure of the system will have on any input, such as the filtering of certain frequency components and the amplification or dampening of the signal. Biological inputs may take many different forms, such as single pulses, slowly decaying signals, constant stimuli applied for a certain time interval, or oscillatory signals. Thus, it is appropriate to have a model in which the output signal is obtained as a convolution of the input $R$ (which may take many forms) and the transfer function $G$ (which depends only on the intrinsic kinase activity parameters and needs to be computed only once).

A very convenient way to analyze system 5 is to convert it to the frequency domain, by application of the Laplace transform operator. The Laplace transform of the impulse response is called the transfer function of the system, and it provides a simple linear relationship between the Laplace transforms of the input and the output, as well as also providing a measure of amplification/dampening of the input signal. The transfer function is given by a simple formula in terms of the matrices $A, B$, and $C$ as summarized in the Supporting Information. For this cascade system, we will carry out the Laplace transforms in detail so as to gain some insight into the internal structure of the system.

The Laplace transform of $X$ will be denoted by $\hat{X}$, and is defined as

$$
\hat{X}_{i}(s)=\int_{-\infty}^{\infty} e^{-s t} X_{i}(t) \mathrm{d} t \quad \hat{R}(s)=\int_{-\infty}^{\infty} e^{-s t} R(t) \mathrm{d} t
$$

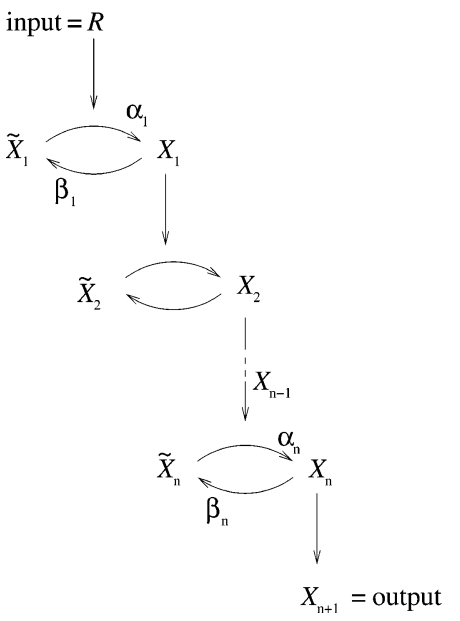

Figure 1. A model of a MAPK cascade.

$$
\stackrel{R}{\rightarrow} G_{1} \stackrel{X_{1}}{\rightarrow} G_{2} \stackrel{X_{2}}{\rightarrow} \ldots \ldots . . X_{i-1}, G_{n} \stackrel{X_{n}}{\rightarrow} G_{n+1} \longrightarrow X_{n+1}
$$

Figure 2. Transfer functions at each step.

where $s$ is a complex number $s=s_{r e}+j \omega \xi$ is the imaginary number $\sqrt{ }-1$ ) and takes values in an appropriate region of convergence.

Applying the Laplace transform operator to both sides of eq 4 , assuming that $X(0)=0$, and recalling the properties of the Laplace transform, we have

$$
\begin{gathered}
s \hat{X}_{1}(s)=\alpha_{1} \hat{R}(s)-\beta_{1} \hat{X}_{1}(s) \\
s \hat{X}_{i}(s)=\alpha_{i} \hat{X}_{i-1}(s)-\beta_{i} \hat{X}_{i}(s) \quad i=2, \ldots, n \\
s \hat{X}_{n+1}(s)=\hat{X}_{n}(s)-l \hat{X}_{n+1}(s)
\end{gathered}
$$

which yields

$$
\hat{X}_{1}(s)=\frac{\alpha_{1}}{s+\beta_{1}} \hat{R}(s) \quad \hat{X}_{i}(s)=\frac{\alpha_{i}}{s+\beta_{i}} \hat{X}_{i-1}(s) \quad i=2, \ldots, n
$$

and

$$
\hat{X}_{n+1}(s)=\frac{1}{s+l} \hat{X}_{n}(s)
$$

In this way, we may view the cascade as a sequence of $n$ steps, the output of the step $i-1$ becoming the input to step $i$.For each single step in the cascade, the input is $\hat{X}_{i-1}$ and the output is $\hat{X}_{i}$, and they are related by a multiplicative factor, which is in fact the transfer function for the step $i$

$$
\hat{G}_{i}(s)=\frac{\alpha_{i}}{s+\beta_{i}} \quad i=2, \ldots, n
$$

For the whole cascade, the input is $R$ and the output is $\hat{X}_{n+1}$, and it is easy to see that the transfer function for the total system is the product of the transfer functions at each step

$$
\hat{G}(s)=\hat{G}_{1}(s) \cdots \hat{G}_{n+1}(s)=\frac{1}{s+l} \frac{\alpha_{1} \cdots \alpha_{n}}{\left(s+\beta_{1}\right) \cdots\left(s+\beta_{n}\right)}
$$

Therefore

$$
\hat{Y}(s)=\hat{X}_{n+1}(s)=\frac{1}{s+l} \frac{\alpha_{1} \cdots \alpha_{n}}{\left(s+\beta_{1}\right) \cdots\left(s+\beta_{n}\right)} \hat{R}(s)
$$

and the actual output may now be obtained by the inverse 
Laplace transform. Alternatively, even without knowing the exact form of the output, that is, the function $Y(t)$, it is still possible to further characterize the properties of the system, through the 2-norm of the functions $\hat{Y}$ and $\hat{R}$. Define the 2-norm of the function $Y$ and the 2-norm of the Laplace transform $\hat{Y}$ by

$$
\begin{aligned}
& \|Y\|_{2}:=\left[\int_{-\infty}^{+\infty}|Y(t)|^{2} \mathrm{~d} t\right]^{1 / 2} \\
& \|\hat{Y}\|_{2}:=\left[\frac{1}{2 \pi} \int_{-\infty}^{+\infty}|\hat{Y}(j \omega)|^{2} d \omega\right]^{1 / 2}
\end{aligned}
$$

with similar expressions holding for $R$ and $\hat{R}$. (Note: from now on, we will assume that the signals are defined only for positive times, that is, $Y(t)=0$ for $t<0$.) The 2 -norm $\|Y\|_{2}$ provides a measure of the strength of the signals (in analogy to the energy of a mechanical system.) Indeed, these norms provide a very convenient way to relate the input and output because, from Parseval's Theorem, the 2-norm of a function equals the 2-norm of its Laplace transform, and therefore

$$
\|\hat{Y}\|_{2}=\|Y\|_{2},\|\hat{R}\|_{2}=\|R\|_{2}
$$

without the need to compute inverse transforms (a very helpful fact, since in general the inverse transforms may not be simple to compute).

Another useful measure is the infinity norm of the transfer function, that selects the least upper bound of the absolute value of $\hat{G}$

$$
\|\hat{G}\|_{\infty}:=\sup _{\omega \in \mathbb{R}}|\hat{G}(j \omega)|
$$

A very useful estimate for characterizing the relative strength of the input and output signals is (see, for instance, refs 17 and 19)

$$
\|Y\|_{2} \leq\|\hat{G}\|_{\infty}\|R\|_{2}
$$

where it is immediately apparent that the infinity norm of the transfer function gives an upper bound for the amplification of the input signal throughout the cascade. Moreover, the infinity norm $\|\hat{G}\|_{\infty}$ is in fact the smallest number that satisfies eq 9 , for all input/output pairs (that is, pairs $(R, Y)$, where $Y$ is the output corresponding to the input $R$ ).

To compute the infinity norm of the transfer function for the whole cascade, note that

$$
\left|\hat{G}_{\dot{j}}(j \omega)\right|^{2}=\frac{\left|\alpha_{i}\right|^{2}}{y \omega+\left.\beta_{i}\right|^{2}} \equiv \frac{\alpha_{i}^{2}}{\omega^{2}+\beta_{i}^{2}} \leq \frac{\alpha_{i}^{2}}{\beta_{i}^{2}} \text { for } \omega \in(-\infty, \infty)
$$

and the equality holds for $\omega=0$. Therefore

$$
\|\hat{G}\|_{\infty}=\frac{1}{l} \frac{\alpha_{1} \cdots \alpha_{n}}{\beta_{1} \cdots \beta_{n}}
$$

A necessary condition for amplification of the signal to occur is that $\|\hat{G}\|_{\infty}>1$. Moreover, since $l$ is essentially an independent parameter, introduced for the purpose of defining a reasonable measure of the output, we can say that amplification of the input signal occurs only if

$$
\alpha_{1} \cdots \alpha_{n}>\beta_{1} \cdots \beta_{n}
$$

Recall that $\alpha_{i} \equiv \tilde{\alpha}_{i} X_{\text {tot }, i}$, where $X_{\text {tot }, i}$ is the total concentration of the $i$ th kinase and $\tilde{\alpha}_{i}$ is the (true) rate of phosphorylation. Therefore, we still expect that $\tilde{\alpha}_{i}<\beta_{i}, i=1, \ldots, n$, as should be the case for a weakly activated pathway.
The norm $\|\hat{G}\|_{\infty}$ is often called the internal gain of the system which, through expression 9, provides a useful and easy way to compute the input-to-output strength relation. For example, if a MAPK cascade has a "5-fold amplification", then its internal gain is $\|\hat{G}\|_{\infty}=5$.

Note that, in the case where $l=0$, the internal gain $\|\hat{G}\|_{\infty}$ is infinite, meaning that in at least one step $\left(X_{n} \rightarrow X_{n+1}\right)$ there is no degradation term. Then the estimate 9 contains no useful information. However, for $l=0$, we have

$$
Y(t)=X_{n+1}(t)=\int_{0}^{t} X_{n}\left(t^{\prime}\right) \mathrm{d} t^{\prime}
$$

and we also have an estimate for the "strength" of the signal $X_{n}$, since

$$
\left\|X_{n}\right\|_{2} \leq \frac{\alpha_{1} \cdots \alpha_{n}}{\beta_{1} \cdots \beta_{n}}\|R\|_{2}
$$

\section{Signaling Time, Signal Duration, and Signal Amplitude}

Some basic quantities which serve to characterize a signal transduction system are the overall amplification from the input to the ouput, the duration of the output signal, and the time it takes the input signal to traverse the cascade. There are several possible definitions and estimates of these quantities; here we extend the definitions given by ref 10, embedding them in the context of frequency-domain analysis and generalizing them to arbitrary inputs.

To be concise, let us identify the cascade 5 by its parameters and associate with it the following $(2 n+1)$-tuple

$$
G:=\left(n, \alpha_{1}, \ldots, \alpha_{n}, \beta_{1}, \ldots, \beta_{n}\right)
$$

where it is assumed that $n \in \mathbb{N}$ and $\alpha_{i}$ and $\beta_{i}$ are positive real numbers, for $i=1, \ldots, n$.

We will also introduce the notation $\mathcal{U}$ for denoting the set of inputs.

Definition 3.1. For system 5, with parameters $C$ and a leak factor $l>0$, for each input $R$, the signaling time, $\tau$, and the output signal duration, $\sigma$, are given by

$$
\left.\left.\tau(\mathcal{G}, l, R):=-\frac{\mathrm{d} \ln \hat{Y}}{\mathrm{~d} s}(s)\right\rfloor_{s=0} \quad \sigma(\mathcal{G}, l, R):=\left(\frac{\mathrm{d}^{2} \ln \hat{Y}}{\mathrm{~d} s^{2}}(s)\right\rfloor_{s=0}\right)^{1 / 2}
$$

The signaling time to step $i$ and the signal duration at step $i, i$ $\leq n$, are given by

$$
\left.\left.\tau_{i}(G, R):=-\frac{\mathrm{d} \ln \hat{X}_{i}}{\mathrm{~d} s}(s)\right\rfloor_{s=0} \quad \sigma_{i}(G, R):=\left(\frac{\mathrm{d}^{2} \ln \hat{X}_{i}}{\mathrm{~d} s^{2}}(s)\right\rfloor_{s=0}\right)^{1 / 2}
$$

To understand the significance of these definitions, recall the properties of the Laplace transform and compute (with $Y(t)=$ 0 for $t \leq 0$ )

$$
\begin{aligned}
& \hat{Y}(0)=\int_{0}^{\infty} Y(t) \mathrm{d} t \frac{\mathrm{d} \hat{Y}}{\mathrm{~d} s}(0)=-\int_{0}^{\infty} t Y(t) \mathrm{d} t \\
& \frac{\mathrm{d}^{2} \hat{Y}}{\mathrm{~d} s^{2}}(0)=\int_{0}^{\infty} t^{2} Y(t) \mathrm{d} t
\end{aligned}
$$

and thus we recover expressions 4 and 5 of ref 10

$$
\tau=\frac{\int_{0}^{\infty} t Y(t) \mathrm{d} t}{\int_{0}^{\infty} Y(t) \mathrm{d} t} \quad \sigma^{2}=\frac{\int_{0}^{\infty} t^{2} Y(t) \mathrm{d} t}{\int_{0}^{\infty} Y(t) \mathrm{d} t}-\left(\frac{\int_{0}^{\infty} t Y(t) \mathrm{d} t}{\int_{0}^{\infty} Y(t) \mathrm{d} t}\right)^{2}
$$




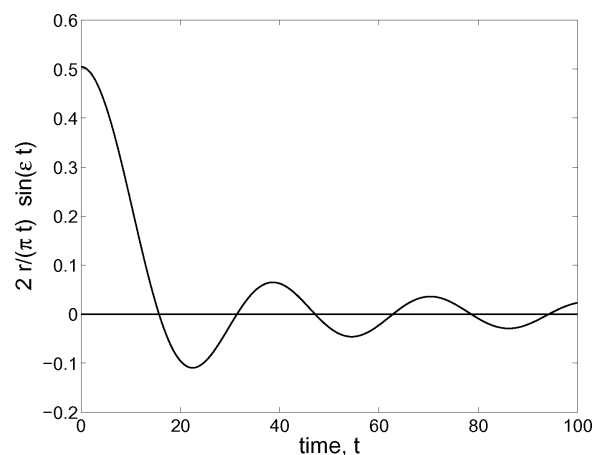

Figure 3. An input that satisfies $\|R\|_{2}=1$ and $\|Y\|_{2} \approx\|\hat{G}\|_{\infty}$, with $\epsilon$ $=0.2$.

where $\tau$ can be regarded as the expected value (of the time to traverse the pathway) and $\sigma$ as the corresponding variance.

An estimate of the amplitude of the output signal, as given in eq 6 of ref 10 , is the value $S$, such that $2 \sigma S=\int_{0}^{\infty} Y(t) \mathrm{d} t$. Again, we propose a more generalized notion, suggested by the input-to-output estimate (eq 9), that takes advantage of the easily computed internal gain of the system, and also incorporates the strength of the signal.

Definition 3.2. For system 5, with parameter $C$ and a leak factor $l>0$, for each input $R$, the signal amplitude is given by

$$
\mathcal{A}(G, l, R):=\frac{\left\|\hat{G}_{G}\right\|_{\infty}}{\sigma(G, l, R)}\|R\|_{2}
$$

where $\hat{G}_{\mathcal{O}}$ is the transfer function of eq $8 . \mathcal{A}$ may also be regarded as the amplitude of a constant signal of duration $\sigma$, but Definition 3.2 differs from the definition of amplitude given in 10 in essentially three points:

1. The meaningful quantity for measuring the amplitude is not the integral $\int Y(t) \mathrm{d} t$ (which computes the area under the curve $Y(t))$ but rather the 2-norm $\left(\int|Y(t)|^{2} \mathrm{~d} t\right)^{1 / 2}$, which computes the strength of the signal.

2. The amplitude is proportional to the product of the internal gain of the system and the 2-norm of the input. This simplifies calculations since, for each cascade, the $\|\hat{G}\|$ is computed only once and $\|R\|_{2}$ is computed for each input signal.

3. The product $\|\hat{G}\|_{\infty}\|R\|_{2}$ is used as an estimate for $\|Y\|_{2}$, but we know that $\|\hat{G}\|_{\infty}$ is the least factor that satisfies the inequality $\|Y\|_{2} \leq \kappa\|R\|_{2}$. In fact, ref 19 shows how to construct examples of inputs for which the equality is approximated. For instance, for any $\epsilon>0$, the input depicted in Figure 3

$$
R(t)=2 \frac{r}{\pi t} \sin \epsilon t \quad \text { with } \quad r=(\pi / \epsilon)^{1 / 2} \text { for } t \geq 0
$$

has unit norm, i.e., $\|R\|_{2}=1$, and satisfies $\|Y\|_{2} \approx\|\hat{G}\|_{\infty}$, for $\epsilon$ small enough, as shown in Supporting Information. (One can also show the existence of positive-valued inputs satisfying this. ${ }^{24}$ )

We remark that these definitions are valid not only for the special case when $A, B$, and $C$ are of the form specified in eqs 6 and 7, but in fact they are valid for any linear system of the form 5. For instance, in section 5, we compute these quantities for the case when there is positive feedback from the last to the first kinase. We next explicitly compute these quantities for the special case when $A, B$, and $C$ are of the forms of eqs 6 and 7 and $l=0$

$$
\left.\tau(G, l, R)=\frac{1}{l}+\sum_{i=1}^{n} \frac{1}{\beta_{i}}+\frac{\mathrm{d} \ln \hat{R}}{\mathrm{~d} s}\right\rfloor_{s=0}
$$

$$
\sigma(G, l, R)=\sqrt{\frac{1}{l^{2}}+\sum_{i=1}^{n} \frac{1}{\beta_{i}^{2}}+q(R)}
$$

where $\left.q(R)=\mathrm{d}^{2} \ln \hat{R} / \mathrm{d} s^{2}\right\rfloor_{s=0}$, and

$$
\mathcal{A}(G, l, R)=\frac{1}{\sqrt{\frac{1}{l^{2}}+\sum_{i=1}^{n} \frac{1}{\beta_{i}^{2}}+q(R)}} \frac{\alpha_{1} \cdots \alpha_{n}}{l \beta_{1} \cdots \beta_{n}}\|R\|_{2}
$$

In the case $l=0$, the quantities $\tau, \sigma$, and $\mathcal{A}$ may be computed for $Y \equiv X_{n}$. The expressions are very similar, except that all the terms in $l$ vanish.

Example 3.3. A typical input is a decaying exponential $R(t)$ $=R_{0} \mathrm{e}^{-\lambda t}$, with

$$
\|R\|_{2}=\frac{R_{0}}{2 \lambda} \quad \hat{R}(s)=\frac{R_{0}}{s+\lambda} \quad \frac{\mathrm{d} \ln \hat{R}}{\mathrm{~d} s}(0)=-\frac{1}{\lambda} \quad q(R)=\frac{1}{\lambda^{2}}
$$

A "peak"-like input may be represented by $R(t)=R_{0} t \mathrm{e}^{-\lambda t}$, with

$$
\|R\|_{2}=\frac{R_{0}}{4 \lambda^{3}} \quad \hat{R}(s)=\frac{R_{0}}{(s+\lambda)^{2}} \quad \frac{\mathrm{d} \ln \hat{R}}{\mathrm{~d} s}(0)=-\frac{2}{\lambda} \quad q(R)=\frac{2}{\lambda^{2}}
$$

For a constant signal, of magnitude $R_{0}$, applied for an interval of time $T_{0}$, we have

$$
\begin{aligned}
& \|R\|_{2}=R_{0} \sqrt{T_{0}} \quad \hat{R}(s)=R_{0} \frac{1-\mathrm{e}^{-s T_{0}}}{s} \\
& \frac{\mathrm{d} \ln \hat{R}}{\mathrm{~d} s}(0)=-\frac{T_{0}}{2} \quad q(R)=\frac{T_{0}{ }^{2}}{12}
\end{aligned}
$$

\section{Cascade Design Optimization}

From the analysis of the quantities $\tau, \sigma$, and $\mathcal{A}$, defined in section 3, we can explore the signaling efficiency of kinase cascades. The definition of an "efficient" response may depend on the particular biological context, but it typically involves the relationship between the length of the cascade, the amplitude of the signal, and its duration. A question posed in ref 10 is whether cascades can respond with sharp signals, i.e., simultaneously of short duration and high amplitude. Our model provides a definite answer to this question.

As we have seen, our linear model has a gain that depends on the length of the cascade and the values of the on/off rate constants but does not depend on the input. As a starting point, we may think of the family of cascades that have the same value for the internal gain, say $K$, and examine their length, the distribution of the "on/off" rates, and signal amplitude and duration. The problem we would like to study is then:

(P) For each fixed internal gain, $\|\hat{G}\|_{\infty}=K$, find the optimal combination of the on/off rates and the length of the cascade that maximizes the signal amplitude, $\mathcal{A}$, for any input $R$.

To formulate this problem, first define the family of cascades that have the same internal gain $K$

$$
\mathbf{C}_{K, l}:=\left\{\mathcal{O}=\left(n, \alpha_{1}, \ldots, \alpha_{n}, \beta_{1}, \ldots, \beta_{n}\right): \frac{\alpha_{1} \cdots \alpha_{n}}{l \beta_{1} \cdots \beta_{n}}=K\right\}
$$

For each input $R$, and each leak factor $l$, define the set of "optimal" cascades, that is, those cascades which exhibit maximal signal amplitude 


$$
\begin{array}{ll}
\mathbf{C}_{\max }(l, R):=\left\{\mathscr{G} \in \mathbf{C}_{K, l}: \mathscr{M}(G, l, R) \geq \mathcal{M}(G, l, R)\right. & \text { for } \\
& \left.G \in \mathbf{C}_{K, l}\right\}
\end{array}
$$

Then define the function

$$
\sigma_{0}\left(n, \beta_{1}, \ldots, \beta_{n}\right):=\sum_{i=1}^{n} \frac{1}{\beta_{i}^{2}}
$$

and observe that it satisfies

$$
\sigma(C, l, R)=\left(\frac{1}{l^{2}}+\sigma_{0}\left(n, \beta_{1}, \ldots, \beta_{n}\right)+q(R)\right)^{1 / 2}
$$

Finally, define the set of cascades that minimize $\sigma_{0}$ over the family $\mathbf{C}_{K, l}$

$$
\begin{array}{r}
\mathbf{C}_{*}(l, R):=\left\{G \in \mathbf{C}_{K, l}: \sigma_{0}\left(n, \beta_{1}, \ldots, \beta_{n}\right) \leq \sigma_{0}\left(n^{\prime}, \beta_{1}^{\prime}, \ldots, \beta_{n}^{\prime}\right) \quad\right. \text { for } \\
\left.G \in \mathbf{C}_{K, l}\right\}
\end{array}
$$

Our first result states that in fact the sets $\mathbf{C}_{*}(l, R)$ and $\mathbf{C}_{\max }(l, R)$ are equal or, in other words, that an optimal cascade will simultaneously maximize the signal amplitude and minimize the signal duration.

Lemma 4.1. In the notation defined above, $\mathbf{C}_{\max }(l, R)=\mathbf{C}_{*-}$ $(l, R)$, for all inputs $R \in U$ and leak factors $l>0$.

The proof is provided in the Supporting Information. An immediate conclusion from this Lemma is that

maximize $\mathscr{U}(\zeta, l, R)$ over $\mathbf{C}_{K, l}$

$\Leftrightarrow$ minimize $\sigma_{0}\left(n, \beta_{1}, \ldots, \beta_{n}\right)$ over $\mathbf{C}_{K, l}$

so that, for any fixed internal gain, maximal amplitude is achieved simultaneously with minimal signal duration. This is consistent with the notion that the most efficient cascade would respond with sharp (high-peaked and fast) output signals. In the limit, this notion can be regarded as an "instantaneous response" $(\sigma \approx 0)$ coupled with "infinite signal amplitude" ( $\mathscr{C}$ $\approx \infty$ ), which is, of course, not biologically viable. A realistic solution to problem $(\mathrm{P})$ does exist and is stated in Theorem 1.

Since the signal duration depends only on the cascade length and the "off" rates, $\beta_{i}$, (besides the input term), we expect the "on" rates, $\alpha_{i}$, to play a small role in maximizing the efficiency of the output response. So, for addressing the problem (P), we will consider two different assumptions on the available knowledge of $\alpha_{i}$ : either (a) all the $\alpha_{i}$ have an equal, fixed value, $\alpha$; or (b) the product of the $\alpha_{i}$ is known, at some fixed $\alpha_{\mathrm{P}}$. We will also assume that the "leak" factor $l$ is fixed, since this parameter was added artificially and may be adjusted independently.

Before stating the main Theorem, we need to introduce some notation. Define the function $f:(1, \infty) \rightarrow(0, \infty)$ to be

$$
f(k)=k^{2}\left[\left(1+\frac{1}{k}\right) \ln \left(1+\frac{1}{k}\right)-\frac{1}{k}\right]
$$

Some properties of this function are stated in the Supporting Information. For any real number $M \geq 1$, define

$$
\begin{gathered}
\lfloor M\rfloor=\text { largest integer less than or equal to } M \\
\lceil M\rceil=\text { least integer greater than } M
\end{gathered}
$$

which are also known as, respectively, the "floor" and "ceiling" functions of $M$. Observing that any real number $M \geq 1$ can be written as the sum of its integral and fractional parts

$$
M=\lfloor M\rfloor+\delta_{M}
$$

where $\delta_{M} \in[0,1)$, define the function $\Psi:(-\infty, \infty) \rightarrow \mathbb{N}$, which is plotted in Figure 4

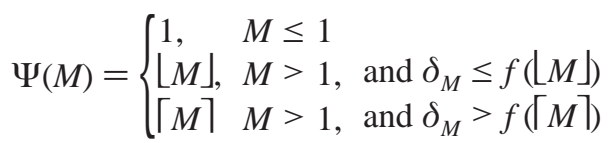

This is a step function where the "jump" between steps always occurs in an interval between two integers, say $k$ and $k+1$, at a point that depends on the number $k$. In particular, since the function $f$ is strictly increasing and takes values in the interval $(2 \ln 2-1,1 / 2)$ (see Supporting Information), it follows that in some cases only the fractional part of the number $M$ affects the location of the "jump" discontinuity

$$
\begin{gathered}
0 \leq \delta_{M}<2 \ln 2-1 \quad \Psi(M)=\lfloor M\rfloor \\
\frac{1}{2}<\delta_{M}<1 \quad \Psi(M)=\lceil M\rceil
\end{gathered}
$$

while for the other cases, $2 \ln 2-1<\delta_{M}<0.5$, the choice depends also on the integral part of $M$.

Theorem 1. Let $K>0$ and $l>0$ be fixed real numbers. Let $\mathbf{C}_{k, l}$ be the set of all cascades (eq 5) with internal gain $K$, as defined above. Then:

1. For each fixed $n=N \in \mathbb{N}$, the elements $O=$ $\left(N, \alpha_{1}, \ldots, \alpha_{N}, \beta_{1}, \ldots, \beta_{N}\right) \in \mathbf{C}_{*}(l, R)$ satisfy $\beta_{i}=\beta$, for all $i=1, \ldots$, $N$, where

$$
\beta=\left(\frac{\alpha_{1} \cdots \alpha_{N}}{K l}\right)^{1 / N}
$$

2 (a). Any element $\mathcal{O} \in \mathbf{C}_{*}(l, R)$ of the form $G=$ $\left(n, \alpha, \ldots, \alpha, \beta_{1}, \ldots, \beta_{n}\right)$ satisfies

$$
n=\Psi(2 \ln K l) \text { and } \quad \beta_{i}=\beta=\alpha\left(\frac{1}{K l}\right)^{1 / n}
$$

2 (b). Any element $G \in \mathbf{C}_{*}(l, R)$ of the form $G=$ $\left(n, \alpha_{1}, \ldots, \alpha_{n}, \beta_{1}, \ldots, \beta_{n}\right) \in \mathbf{C}_{*}(l, R)$ with $\alpha_{1} \cdots \alpha_{n}=\alpha_{p}$ satisfies

$$
n=\Psi\left(2 \ln \frac{K l}{\alpha_{\mathrm{p}}}\right) \text { and } \beta_{i}=\beta=\left(\frac{\alpha_{\mathrm{p}}}{K l}\right)^{1 / n}
$$

Before presenting the proof of the Theorem, some remarks on the interpretation of points $1,2 \mathrm{a}$, and $2 \mathrm{~b}$. The first part of the result is consistent with the observation that the ordering of the amplification or dampening single steps within the cascade does not influence the final output signal (also observed in ref 10).

The second part of the Theorem shows that indefinitely increasing the cascade's length will not increase amplification. In fact, there is an optimal length for the cascade that provides both maximum signal amplitude and minimum duration. A similar observation was mentioned in ref 10, and our Lemma 4.1 and Theorem 1 characterize the conditions for achieving this optimization. For each gain $K$ and leak factor $l$, this optimal length is easily read out from Figure 4 . For instance, a cascade with a $6-9$-fold gain (and $l=1$ ) is seen to have an optimal length of 4 steps. Figure 5 illustrates Theorem 1, for an 8-fold cascade gain. The Figure shows the results of two simulations of system 5 , both with input $R(t)=5 t \mathrm{e}^{-2 t}$ but different lengths of the cascade. The various curves represent $R$, the concentrations of each kinase $X_{i}, i=1, \ldots, n$, and the output $X_{n+1}$. It is clear that, for the nonoptimal $n=7$, the output's amplitude 

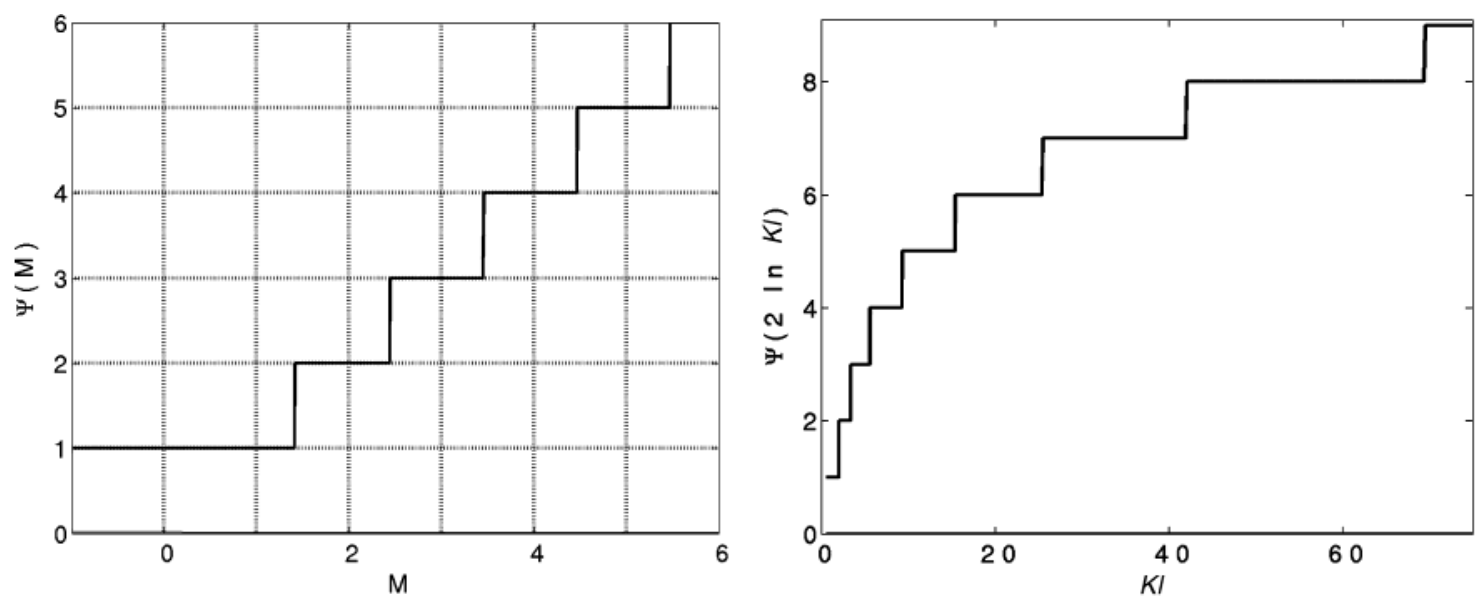

Figure 4. Left: the function $\Psi(M)$. Right: the function $\Psi(2 \ln K l)$. Note that, for a given gain $K$ and leak factor $l$, the optimal length is given by the integer platform corresponding to the product $K l$.
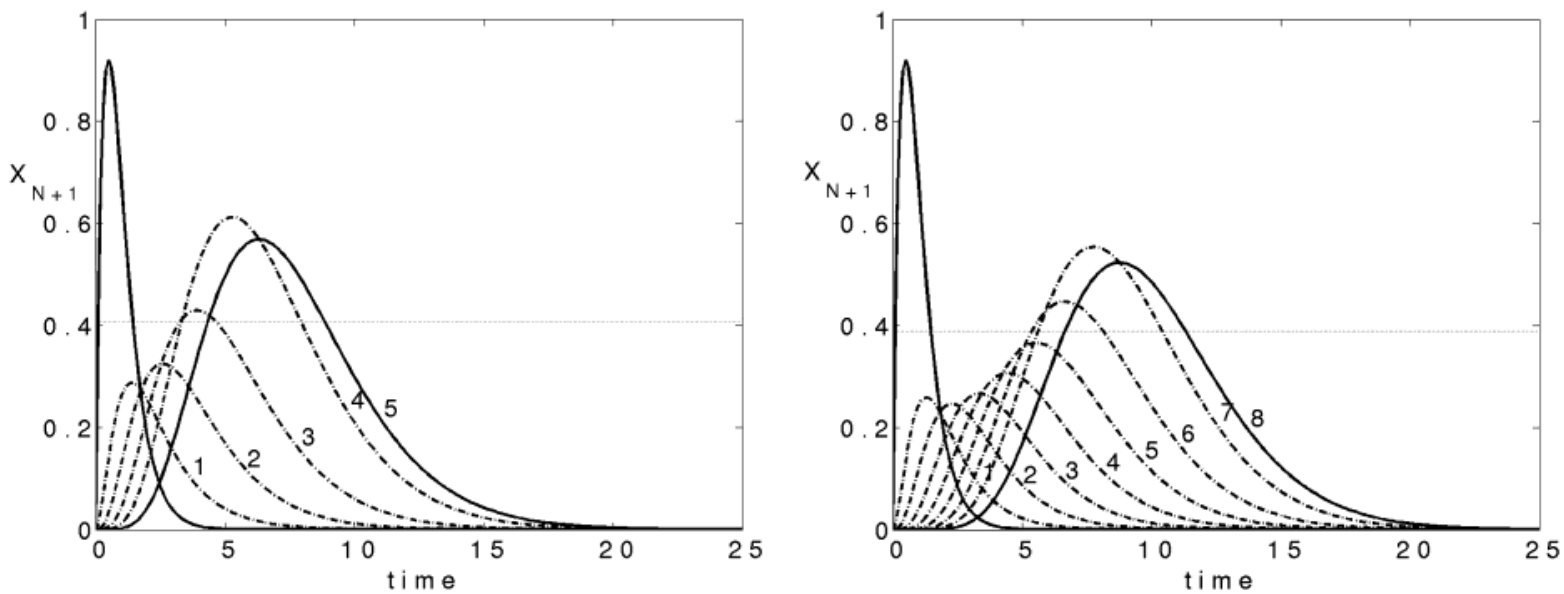

Figure 5. Signal transduction cascade with $R(t)=5 t \mathrm{e}^{-2 t}$, with $K=8, l=1, \alpha_{i}=1.2$. The horizontal lines represent $\mathcal{A}$. Left (optimal case): $n$ $=4, \beta_{i} \approx 0.714, i=1, \ldots, n, \mathcal{A}=0.409$, and $\sigma_{0}=3.059$. Right: $n=7, \beta_{i} \approx 0.892, i=1, \ldots, n, \mathcal{A}=0.389$, and $\sigma_{0}=3.210$.

decreases and the signal duration increases. Note that the output curve $X_{8}$ is more spread out across time and its maximum value is lower, than for the optimal case.

Theorem 1 can be proved by successively solving the two optimization problems:

(P1) For each fixed $n$, minimize $\sigma_{0}$, over all possible choices of $\beta_{1}, \ldots, \beta_{n} \in(0, \infty)$, subject to $\|\hat{G}\|_{\infty}=K$, and

(P2) Minimize $\sigma_{0}$, over all possible choices of $n \in \mathbb{N}$ and $\beta_{1}$, $\ldots, \beta_{n} \in(0, \infty)$, subject to $\|\hat{G}\|_{\infty}=K$. Recall that we are assuming that either (a) all the $\alpha_{i}$ have an equal, fixed value, $\alpha$, or (b) the product of the $\alpha_{i}$ value is known, at some fixed $\alpha_{\mathrm{P}}$. The solution of (P1) is equal for both cases, but the solution of (P2) is slightly different for a or b. Thus, problem (P1) is part 1 and (P2) is the part 2 of the Theorem. As we will see, the solution of (P1) greatly simplifies the proof of (P2).

4.1. Solving (P1): Proof of Part 1 of Theorem 1. Given a cascade of length $n$, this problem consists of finding a set of $n$ parameters $\bar{\beta}_{1}, \ldots, \bar{\beta}_{n}$ such that the function $\sigma_{0}$ attains a minimum value at $\bar{\beta}_{i}, i=1, \ldots, n$, i.e.

$$
\frac{1}{\bar{\beta}_{1}^{2}}+\frac{1}{\bar{\beta}_{2}^{2}}+\cdots+\frac{1}{\bar{\beta}_{n-1}{ }^{2}} \leq \frac{1}{\beta_{1}{ }^{2}}+\frac{1}{\beta_{2}^{2}}+\cdots+\frac{1}{\beta_{n-1}{ }^{2}}
$$

for every $\beta_{1}, \ldots, \beta_{n}$ such that $\|\hat{G}\|_{\infty}=K$

$$
\|\hat{G}\|_{\infty}=\frac{1}{l} \frac{\alpha_{1} \cdots \alpha_{n}}{\beta_{1} \cdots \beta_{n}}=K \Leftrightarrow \mathrm{K} l \beta_{1} \cdots \beta_{n}-\alpha_{1} \cdots \alpha_{n}=0
$$

For simplicity, rescale the values to $B_{i}=1 / \beta_{i}^{2}$, and observe that

$$
\frac{1}{B_{1} \cdots B_{n}}=\left(\beta_{1} \cdots \beta_{n}\right)^{2}=\left(\frac{\alpha_{1} \cdots \alpha_{n}}{K l}\right)^{2}
$$

Then, the problem consists of minimizing the function

$$
F\left(B_{1}, \ldots, B_{n-1}\right)=B_{1}+\cdots+B_{n-1}+\frac{Q}{B_{1} \cdots B_{n-1}}
$$

over all possible choices of $B_{i}>0, i=1, \ldots, n-1$, where

$$
Q=\left(\frac{K l}{\alpha_{1} \cdots \alpha_{n}}\right)^{2}
$$

In the Supporting Information, we show that the solution to this optimization problem is

$$
B_{i}=Q^{(1 / n)} \quad i=1, \ldots, n-1
$$

which also implies

$$
B_{n}=\frac{Q}{Q^{(1-1 / n)}}=Q^{(1 / n)}
$$

So, the choice of the "off" rate constants that minimizes $\sigma_{0}$ is to have $\beta_{1}=\beta_{2}=\cdots=\beta_{n}=\bar{\beta}$, with 


$$
\bar{\beta}=\frac{1}{\sqrt{B_{n}}}=\left(\frac{\alpha_{1} \cdots \alpha_{n}}{K l}\right)^{(1 / n)}
$$

as we wanted to show.

4.2. Solving (P2): Proof of Part 2 of Theorem 1. To solve the more general problem, we first show how its statement can be simplified. Given the value of $\alpha$ (respectively, $\alpha_{P}$ ), suppose that we have found a solution of (P2), i.e., an integer $n^{*}$ and a set of constants $\beta_{i}^{*}, i=1, \ldots, n^{*}$ satisfying

$$
\sigma_{0}\left(n^{*}, \beta_{1}^{*}, \ldots, \beta_{n^{*}}^{*}\right) \leq \sigma_{0}\left(n, \beta_{1}, \ldots, \beta_{n}\right)
$$

for any other cascade $C=\left(n, \alpha_{1}, \ldots, \alpha_{n}, \beta_{1}, \ldots, \beta_{n}\right)$ with $\alpha_{i}=$ $\alpha, i=1, \ldots, n$ (respectively, $\alpha_{1} \cdots \alpha_{n}=\alpha_{P}$ ).

We have already showed that

$$
\sigma_{0}\left(n^{*}, \bar{\beta}^{*}, \ldots, \bar{\beta}^{*}\right) \leq \sigma_{0}\left(n^{*}, \beta_{1}^{*}, \ldots, \beta_{n^{*}}^{*}\right)
$$

with

$$
\bar{\beta}^{*}=\left(\frac{\alpha_{1} \cdots \alpha_{n^{*}}}{K l}\right)^{\left(1 / n^{*}\right)}
$$

and we know this choice yields the unique minimum of $\sigma_{0}$ for a fixed length $n$. So, it follows that the solution of (P2) must also satisfy

$$
\beta_{i}^{*}=\bar{\beta}^{*} \quad i=1, \ldots, n^{*}
$$

This observation allows us to simplify the statement of problem P2 and look only for solutions where all $\beta_{i}$ values are equal: (P2) ${ }^{\prime}$ Minimize $\sigma_{0}(n, \beta, \ldots, \beta)=n / \beta^{2}$, over $n \in \mathbb{N}$ and $\beta \in(0, \infty)$, subject to $(\alpha / \beta)^{n}=K l$.

From the constraint $\|\hat{G}\|_{\infty}=K$, we have

Case 2a:

$$
\left(\frac{\alpha}{\beta}\right)^{n}=K l \Leftrightarrow \beta=\alpha\left(\frac{1}{K l}\right)^{(1 / n)} \Longrightarrow \sigma_{0}(n, \beta(n))=\frac{1}{\alpha^{2}} n(K l)^{(2 / n)}
$$

Case 2b:

$$
\frac{\alpha_{\mathrm{P}}}{\beta^{n}}=K l \Leftrightarrow \beta=\left(\frac{\alpha_{\mathrm{P}}}{K l}\right)^{(1 / n)} \Longrightarrow \sigma_{0}(n, \beta(n))=n\left(\frac{K l}{\alpha_{\mathrm{P}}}\right)^{(2 / n)}
$$

In either case, to solve the problem, it is enough to minimize the function $\ln \left[\sigma_{0}(n, \beta(n))\right]$

$$
F(n, M)=\ln n+\frac{1}{n} M
$$

over $n \in \mathbb{N}$, where $M$ is a positive constant with value either

$$
\begin{aligned}
& M=2 \ln \mathrm{K} l, \text { for case } 2 \mathrm{a} \\
& M=2 \ln \frac{K l}{\alpha_{\mathrm{P}}}, \text { for case } 2 \mathrm{~b}
\end{aligned}
$$

For a fixed $M$, let the minimizer of $F(n, M)$ over $n \in \mathbb{N}$ be

$$
n^{*}(M):=\left\{n \in \mathbb{N}: F(n, M) \leq F\left(n^{\prime}, M\right) \text { for } \quad n^{\prime} \in \mathbb{N}\right\}
$$

which is given by (see the Supporting Information for a proof)

$$
n^{*}(M)=\Psi(M)
$$

Thus, for part 2a of the Theorem, we have $n=n^{*}(2 \ln K l)$ $=\Psi(2 \ln K l)$, and for part $2 \mathrm{~b}$, we have $n=\Psi\left(2 \ln K l / \alpha_{\mathrm{P}}\right)$. The value $\beta$ is given according to part 1 .
As shown in the example of Figure 6, evaluation of $\sigma_{0}$ at $n^{*}(M)$ yields a value which is actually quite close to the "true" $\sigma_{0}(M, \beta(M))$.

\section{Cascades with Positive Feedback}

In this section, we investigate the behavior of cascades under positive feedback (for a computational study of feedback effects see, for instance, ref 6). Assume that the last kinase, $X_{n}$, also contributes to the activation of the first kinase; then the differential equation for $X_{1}$ includes one more term and becomes

$$
\frac{\mathrm{d} X_{1}}{\mathrm{~d} t}=\alpha_{1} R(t)+\epsilon X_{n}-\beta_{1} X_{1}
$$

We will assume that $\epsilon$ is small enough

$$
\beta_{1} \cdots \beta_{n}>\epsilon \alpha_{2} \cdots \alpha_{n}
$$

This guarantees that the cascade is stable with respect to small perturbations (that is, all the eigenvalues of the system's matrix $A$ have negative real parts, see section 6 ).

We can compute the transfer function for the system with feedback $(\epsilon>0)$, just as we did in section 2, for a given cascade $C=\left(n, \alpha_{1}, \ldots, \alpha_{n}, \beta_{1}, \ldots, \beta_{n}\right)$ and any input $R$ and leak factor $l>0$. We obtain

$$
\hat{G}(s)=\frac{1}{s+l} \frac{\alpha_{1} \cdots \alpha_{n}}{\left(s+\beta_{1}\right) \cdots\left(s+\beta_{n}\right)-\epsilon \alpha_{2} \cdots \alpha_{n}}
$$

The infinity norm is again obtained for the case $\omega=0(s=$ $j \omega)$ (see below, at the end of this section)

$$
\|\hat{G}\|_{\infty}=\frac{1}{l} \frac{\alpha_{1} \cdots \alpha_{n}}{\beta_{1} \cdots \beta_{n}-\epsilon \alpha_{2} \cdots \alpha_{n}}
$$

Computing the signaling time $(\tau)$ and the signal duration $(\sigma)$ and amplitude $(\mathscr{C})$, we have

$$
\left.\tau_{\mathrm{fb}}(G, l, R)=\frac{1}{l}+\frac{\beta_{1} \cdots \beta_{n} \sum_{i=1}^{n} \frac{1}{\beta_{i}}}{\beta_{1} \cdots \beta_{n}-\epsilon \alpha_{2} \cdots \alpha_{n}}+\frac{\mathrm{d} \ln \hat{R}}{\mathrm{~d} s}\right\rfloor_{s=0}
$$

$$
\begin{gathered}
\int_{\mathrm{fb}}(G, l, R)= \\
l^{2}+\frac{\left(\beta_{1} \cdots \beta_{n}\right)^{2}\left[\sum_{i=1}^{n} \frac{1}{\beta_{i}^{2}}+\epsilon \alpha_{2} \cdots \alpha_{n} \sum_{i \neq j} \frac{1}{\beta_{i} \beta_{j}}\right]}{\left(\beta_{1} \cdots \beta_{n}-\epsilon \alpha_{2} \cdots \alpha_{n}\right)^{2}}+q(R) \\
\mathcal{L}_{\mathrm{fb}}(G, l, R)=\frac{1}{\sigma(G, l, R)} \frac{\alpha_{1} \cdots \alpha_{n}}{\beta_{1} \cdots \beta_{n}-\epsilon \alpha_{2} \cdots \alpha_{n}}\|R\|_{2}
\end{gathered}
$$

Comparison of these quantities for the models with and without feedback leads to the following conclusions:

1. The system with feedback exhibits higher internal gain.

2. The system with feedback exhibits larger signaling time and signal duration $\tau_{\mathrm{fb}}>\tau$ and $\sigma_{\mathrm{fb}}>\sigma$.

So, for an arbitrary cascade, the existence of a positive feedback leads to a less sharp output signal; the signal transduction down the cascade takes a longer time, and the output signal has greater duration.

On the other hand, the existence of feedback may be used to great advantage in the design of an optimal cascade: positive feedback (at a constant rate $\epsilon$ ) allows the cascade to be of shorter 

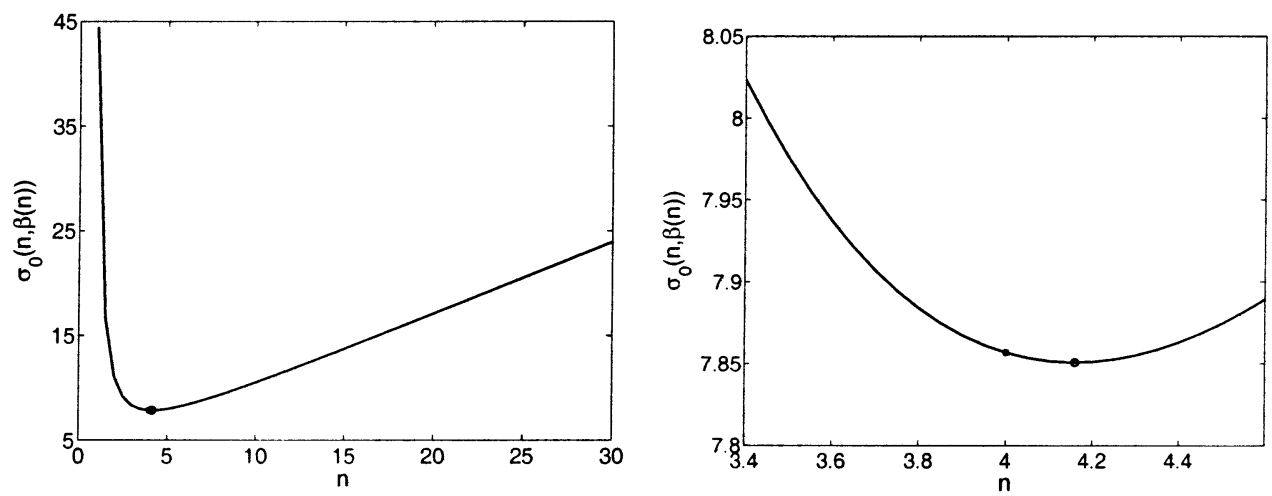

Figure 6. The function $\sigma_{0}(n, \beta(n))$, for $K l=8$ and $\alpha=1.2$, and the points $\left(M, \sigma_{0}(M, \beta(M))\right)$ (circle) and $\left(n^{*}, \sigma_{0}\left(n^{*}, \beta\left(n^{*}\right)\right)\right)$ (star).

length and still have the same maximal amplitude/minimal duration. The results in Theorem 1 are valid just as before, with suitable adjustments to some of the constants. Thus, we now have

$$
\|\hat{G}\|_{\infty}=K \Leftrightarrow \beta_{1} \cdots \beta_{n}=\frac{\left(\alpha_{1}+\epsilon K l\right) \alpha_{2} \cdots \alpha_{n}}{K l}
$$

and now, similarly to the proof in section 4.1 , we set

$$
Q_{\mathrm{fb}}=\left(\frac{K l}{\left(\alpha_{1}+\epsilon K l\right) \alpha_{2} \cdots \alpha_{n}}\right)^{2}
$$

which leads to the optimal value for $\beta_{i}=\beta_{\mathrm{fb}}, i=1, \ldots, n$

$$
\beta_{\mathrm{fb}}=\left(\frac{\left(\alpha_{1}+\epsilon K l\right) \alpha_{2} \cdots \alpha_{n}}{K l}\right)^{(1 / n)}
$$

To find the optimal length of the cascade with feedback, note that

$$
\begin{aligned}
& \sigma_{0}(n, \beta(n))=n M_{\mathrm{fb}}^{(1 / n)} \text { with } \\
& \qquad M_{\mathrm{fb}}=2 \ln \left(\frac{K l}{\left(\alpha_{1}+\epsilon K l\right) \alpha_{2} \cdots \alpha_{n}}\right)^{(1 / n)}
\end{aligned}
$$

Since $M_{\mathrm{fb}} \leq M$, then also $n_{\mathrm{fb}}^{*}\left(M_{\mathrm{fb}}\right) \leq n^{*}(M)$. Therefore, we conclude that, for the cascade with feedback:

3 . For each fixed $n$, the value of the off rates that maximizes $\mathcal{A}\left(\right.$ minimizes $\left.\sigma_{0}\right)$ over $\mathbf{C}_{*}(l, R)$ is larger, $\beta_{\mathrm{fb}}>\beta$.

4. The length of the cascade that maximizes $\mathcal{A}$ (minimizes $\left.\sigma_{0}\right)$ over $\mathbf{C}_{*}(l, R)$ is smaller, $n_{\mathrm{fb}}^{*}<n^{*}$.

These results agree with what would be expected from a signaling pathway: indeed, the existence of positive feedback enhances the activation at each step, so a larger amount of the phosphorylated kinase will be produced; to keep this amount at a "weak" level, the phosphatases should increase their activity. On the other hand, since the amount of phosphorylated kinases increased, a smaller number of steps is required to produce the same signal amplitude as in the cascade with no feedback.

To compute the infinity norm $\|\hat{G}\|_{\infty}$, we first note that the denominator of $\hat{G}(j \omega)$, which we will denote by $\operatorname{den}(\hat{G}(j \omega))$, satisfies (by the triangle inequality)

$$
|\operatorname{den}(\hat{G}(j \omega))| \geq j \omega \omega+l \mid\left[\left|j \omega+\beta_{n}\right| \cdots\left|j \omega+\beta_{n}\right|-\epsilon \alpha_{2} \cdots \alpha_{n}\right]
$$

Also

$$
\begin{aligned}
& j \omega+\beta_{n}\left|\cdots j \omega+\beta_{n}\right|= \\
& \left(\left(\omega^{2}+\beta_{1}{ }^{2}\right) \cdots\left(\omega^{2}+\beta_{n}{ }^{2}\right)\right)^{1 / 2} \geq \beta_{1} \cdots \beta_{n}
\end{aligned}
$$

for every $\omega \in \mathbb{R}$, where the equality holds if and only if $\omega=0$. Thus

$$
|\operatorname{den}(\hat{G}(j \omega))| \geq l\left[\beta_{1} \cdots \beta_{n}-\epsilon \alpha_{2} \cdots \alpha_{n}\right]=\operatorname{den}(\hat{G}(0))>0
$$

where the last inequality follows from the assumption $\beta_{1} \cdots \beta_{n}$ $>\epsilon \alpha_{2} \cdots \alpha_{n}$. Therefore, if the expression $|\operatorname{den}(\hat{G}(j \omega))|$ is minimized at $\omega=0$, then the function $|\hat{G}(j \omega)|$ is maximized at $\omega=$ 0 , as we wanted to show.

\section{Stability of Cascades}

A signaling pathway is considered stable (see ref 10) if small and random perturbations (those that do not consist of biologically relevant inputs) are not amplified. So, in the presence of small perturbations, the amount of phosphorylated kinases should not be allowed to grow very large and should return to the stable state, with $X_{i} \approx 0$, for all $i=1, \ldots, n$. Thus, the behavior of a signaling pathway in the absence of a relevant input always satisfies expression 3 , that is, $X_{i} \ll X_{\text {tot, } i}$ for each $i=1, \ldots, n$, and hence its stability may be established by analysis of model 5 .

In the absence of an input $(R(t) \equiv 0)$, the point $\left(X_{1}, X_{2}, \ldots\right.$, $\left.X_{n+1}\right)=(0,0, \ldots, 0):=\mathbf{0}$ is an equilibrium point of system 5 , and the stability of this equilibrium determines the stability of the pathway. The equilibrium point $\mathbf{0}$ is stable if all the eigenvalues of the matrix $A$ have negative real parts. This is indeed the case for the system described by eq 5 . We know that, after a perturbation, the system will always return to $\mathbf{0}$. Moreover, we can estimate that a small perturbation will also generate a small response, since

$$
\left\|Y_{\text {pert }}\right\|_{2} \leq \kappa\left\|R_{\text {pert }}\right\|_{2}
$$

where $\kappa$ is a constant, equal to $\|\hat{G}\|_{\infty}$.

For signaling cascades that exhibit a lower degree of kinase specificity, the problem of stability of the cascade (see ref 10) becomes significant. If a kinase $X_{i}$ affects both the downstream kinases and some upstream kinase, then the eigenvalues of $A$ change, and stability is not guaranteed. Allowing for kinase nonspecificity, a resulting matrix $A$ could be of the form

$$
A_{\epsilon}=\left(\begin{array}{lllllll}
-\beta_{1} & \epsilon_{12} & 0 & \cdots & 0 & \epsilon_{1 n} & 0 \\
\alpha_{2} & -\beta_{2} & 0 & \cdots & 0 & 0 & 0 \\
\epsilon & \alpha_{3} & -\beta_{3} & \cdots & 0 & 0 & 0 \\
\epsilon & \epsilon & \alpha_{4} & \cdots & 0 & 0 & 0 \\
\vdots & & & \ddots & & \vdots & \\
\epsilon & \epsilon & \epsilon & \cdots & \alpha_{n} & -\beta_{n} & 0 \\
0 & 0 & 0 & \cdots & 0 & 1 & l
\end{array}\right)
$$


and the signaling pathway is stable if all the eigenvalues of $\mathrm{A}_{\epsilon}$ have negative real parts.

Some relevant easy-to-compute examples are:

(1) Suppose that each kinase $i$ is only allowed to activate its downstream kinases $\left(\epsilon_{1 n}=0, \epsilon_{12}=0\right.$, and $\left.\epsilon \neq 0\right)$; in this case, it is not surprising that stability is not affected at all, because this situation corresponds to a lower triangular matrix, again with eigenvalues $-\beta_{i}$;

(2) Suppose that there exists feedback from the last activated kinase to the first kinase $\left(\epsilon_{1 n}=\epsilon_{0}, \epsilon_{12}=0\right.$, and $\left.\epsilon=0\right)$; in this case, if $\epsilon_{0}$ satisfies

$$
\beta_{1} \cdots \beta_{n}>\epsilon_{0} \alpha_{2} \cdots \alpha_{n} \Leftrightarrow \epsilon_{0}<\frac{\beta_{1} \cdots \beta_{n}}{\alpha_{2} \cdots \alpha_{n}}
$$

then the eigenvalues of the matrix $A_{\epsilon}$ all have negative real parts and the new cascade is stable. To prove this, suppose that there exists an eigenvalue of $A_{\epsilon}$ with positive real part, that is, a complex number $\lambda$ such that

$$
\lambda=\lambda_{r e}+j \lambda_{i m} \quad \lambda_{r e} \geq 0
$$

and

$$
\operatorname{det}\left(A_{\epsilon}-\lambda I\right)=(-1)^{n} l\left[\left(\lambda+\beta_{1}\right) \cdots\left(\lambda+\beta_{n}\right)-\epsilon_{0} \alpha_{2} \cdots \alpha_{n}\right]=0
$$

Then eq 25 implies $\left|\lambda+\beta_{i}\right| \geq \beta_{i}$, for $i=1, \ldots, n$ and so

$$
\begin{aligned}
& \left|\left(\lambda+\beta_{1}\right) \cdots\left(\lambda+\beta_{n}\right)-\epsilon_{0} \alpha_{2} \cdots \alpha_{n}\right| \\
& \geq\left|\left(\lambda+\beta_{1}\right) \cdots\left(\lambda+\beta_{n}\right)\right|-\epsilon_{0} \alpha_{2} \cdots \alpha_{n} \\
& \geq \beta_{1} \cdots \beta_{2}-\epsilon_{0} \alpha_{2} \cdots \alpha_{n}>0
\end{aligned}
$$

which contradicts eq 26.

\section{Discussion and Conclusions}

By modeling weakly activated signal transduction cascades as linear systems and applying techniques from control systems theory, one can identify the cascade's input-to-output transfer function and internal gain. On the basis of these properties, the concepts of signal duration, signaling time, and signal amplitude may be defined in an intuitive and general form for any input signal.

Our analysis shows that, for linear cascades, signal amplitude and duration are, respectively, maximized and minimized simultaneously. So, a cascade can respond with signals that are both fast and exhibit high amplification. To achieve the highest amplification and the shortest duration response, the cascade should have all off rates equal to some value $\beta$.

We also show that, for each fixed internal gain, there are finite values for the length of the cascade and the off constants that simultaneously maximize (minimize) the signal amplitude (signal duration). To achieve these optimal conditions, the optimal length should be given by the well-defined step function $\Psi$. This function $\Psi$ depends only on, and increases logarithmically with, the internal gain of the system. The off constants should all have the same value $\beta$. This optimal value $\beta$ depends on the internal gain and the length of the system.

In addition, our analysis shows that a positive feedback term on the cascade enhances the optimal design, by allowing the same signal amplitude and duration to be achieved with a shorter length and higher off rates.
Other issues, such delay at each phosphorylation step, and the stability of the signaling pathway when there is a high degree of nonspecificity among the kinases, are also naturally examined within this framework. We have seen that the stability of the zero steady-state of the cascade with respect to small perturbations is established by checking that the eigenvalues of the matrix $A$ all have negative real parts. Supposing that, at each step, there is a delay $\delta_{i}$ in the transmission of the signal the dynamics at step $i$ becomes $\left(\mathrm{d} X_{i}(t) / \mathrm{d} t\right)=\alpha_{i} X_{i-1}\left(t-\delta_{i-1}\right)-$ $\beta_{i} X_{i}\left(t-\delta_{i}\right)$. The Laplace transform of $X_{i}\left(t-\delta_{i}\right)$ is $\mathrm{e}^{-s \delta_{i}} \hat{X}_{i}$ so that the transfer function becomes

$$
\hat{G}(s)=\frac{1}{s+l} \frac{\alpha_{1} \cdots \alpha_{n}}{\left(s+\beta_{1}\right) \cdots\left(s+\beta_{n}\right)} \mathrm{e}^{-s \delta_{1}} \mathrm{e}^{s \delta_{n+1}}
$$

But, for an imaginary number $j \omega,\left|\mathrm{e}^{-j \omega \delta_{i}}\right|=1$, so the norm $\| \hat{G}||_{\infty}$ is unchanged, and since $\mathrm{e}^{-s \delta_{i}}=1$ when evaluated at $s=0$, the signal duration and amplitude are also unchanged. This is not surprising, because in a linear system, delay simply causes a temporal translation of the signal, by a fixed amount, without affecting amplitudes.

Our analysis has been entirely linear, since we considered only the weakly activated case. The key tool for our analysis was the " $H_{\infty}$ norm" $\|\hat{G}\|_{\infty}$ of the transfer function of the system. Defined in this fashion, this notion only makes sense for linear systems. However, an equivalent definition of $\|\hat{G}\|_{\infty}$ is obtained (see Supporting Information) by considering the smallest constant $c>0$ such that $\|Y\|_{2} \leq c\|R\|_{2}$, that is to say, the induced operator $f^{2}$ norm of the system. This latter characterization is valid for arbitrary nonlinear systems. Recall that we have the following set of equations

$$
\begin{gathered}
\frac{\mathrm{d} X_{1}}{\mathrm{~d} t}=\alpha_{1} R\left(1-\frac{X_{1}}{X_{\mathrm{tot}, 1}}\right)-\beta_{1} X_{1} \\
\frac{\mathrm{d} X_{i}}{\mathrm{~d} t}=\alpha_{i} X_{i-1}\left(1-\frac{X_{i}}{X_{\mathrm{tot}, i}}\right)-\beta_{i} X_{i} \quad i=2, \ldots, n \\
\frac{\mathrm{d} X_{n+1}}{\mathrm{~d} t}=X_{n}-l X_{n+1}
\end{gathered}
$$

and we had simplified (changing notations to use " $Y$ " for future reference) in the case of weakly activated pathways to

$$
\begin{gathered}
\frac{\mathrm{d} Y_{1}}{\mathrm{~d} t}=\alpha_{1} R-\beta_{1} Y_{1} \\
\frac{\mathrm{d} Y_{i}}{\mathrm{~d} t}=\alpha_{i} Y_{i-1}-\beta_{i} Y_{i} \quad i=2, \ldots, n \\
\frac{\mathrm{d} Y_{n+1}}{\mathrm{~d} t}=Y_{n}-l Y_{n+1}
\end{gathered}
$$

From the form of the equations, it is clear that, if $R(t) \geq 0$ for all $t$ and if initial conditions are nonnegative, then solutions of both systems remain nonnegative.

Lemma 7.1. Pick any non-negative input function $R(t)$ and consider any solution of the $X$-system with a nonnegative initial condition $X(0)$ and the solution of the $Y$ system with the same initial condition $Y(0)=X(0)$. Then, $X_{i}(t) \leq Y_{i}(t)$ for all $t \geq 0$ and all $i$.

Proof. By induction on $i$, it is enough to show the following fact: if $p$ is a non-negative constant and $0 \leq u(t) \leq v(t)$ for all $t \geq 0$, then the solution of 


$$
\begin{gathered}
\frac{\mathrm{d} x}{\mathrm{~d} t}=\alpha u(1-p x)-\beta x \\
\frac{\mathrm{d} y}{\mathrm{~d} t}=\alpha v-\beta y
\end{gathered}
$$

with any initial conditions $x(0)=y(0) \geq 0$ satisfies $x(t) \leq y(t)$ for all $t \geq 0$. Let us prove this next. We observe that

$$
\frac{\mathrm{d} x}{\mathrm{~d} t} \leq \alpha u(1-p x)-\beta x \leq \alpha u-\beta x \leq \alpha v-\beta x
$$

because $-p u x \leq 0$ and $u \leq v$. The standard comparison lemma (see, e.g., ref 20, Lemma 2.5) for scalar differential equations says that if $y$ is a solution of a differential equation $\mathrm{d} y / \mathrm{d} t=$ $f(t, y)$ and if $x$ satisfies $\mathrm{d} x / \mathrm{d} t \leq f(t, x(t))$ and $x(0) \leq y(0)$, then $x(t) \leq y(t)$ for all $t$; we simply apply this comparison lemma with $f(t, a)=\alpha v-\beta a$.

Remark 7.2. Instead of using induction on the cascade, the same result could be proved as a consequence of comparison theorems for "monotone" systems with inputs. ${ }^{21}$

As a corollary of the Lemma, we conclude that, for zero initial conditions on the $X_{i}$ values, the $f^{2}$ norm of $X_{n+1}$ satisfies

$$
\left\|X_{n+1}\right\|_{2} \leq\left\|Y_{n+1}\right\|_{2} \leq\|\hat{G}\|_{\infty}\|R\|_{2}
$$

as well. In other words, unless further information is provided regarding the total amounts $X_{\mathrm{tot}, i}$, the calculated gain is also the best possible estimate of the gain for the fully nonlinear system. Of course, if one knows the value of $X_{\mathrm{tot}, i}$, then the calculation of the exact value of the induced $f$ gain (smaller than the estimate given by the linearized system) is a harder problem. Another paper ${ }^{24}$ deals with that study, based upon techniques from "nonlinear $H_{\infty}$ " theory ${ }^{22}$ and more generally, input to state stability theory. ${ }^{23}$

To give a specific example, consider the simplest case of a one step cascade

$$
\begin{gathered}
\frac{\mathrm{d} X_{1}}{\mathrm{~d} t}=\alpha_{1} R\left(1-\frac{X_{1}}{X_{\mathrm{tot}, 1}}\right)-\beta_{1} X_{1} \\
\frac{\mathrm{d} X_{2}}{\mathrm{~d} t}=X_{1}-l X_{2}
\end{gathered}
$$

For inputs of the form $R(t)=R_{0}$ for $0 \leq t \leq T$, and $R(t)=0$ for $t>T$, one can compute the exact value of the $f$ gain, for which we will try to produce a suitable lower bound. Some algebra shows that

$$
\lim _{T \rightarrow \infty} \frac{\left\|X_{2}\right\|_{2}}{\|R\|_{2}}=\frac{1}{l} \frac{\alpha_{1} X_{\mathrm{tot}, 1}}{\beta_{1} X_{\mathrm{tot}, 1}+\alpha_{1} R_{0}}
$$

The largest value of this quotient is obtained for small $R_{0}$. The limiting value as $R_{0} \rightarrow 0$ provides a lower bound for the $f^{2}$ gain

$$
c \geq \frac{1}{l} \frac{\alpha_{1}}{\beta_{1}}
$$

On the other hand, the upper bound on $c$ is, using Lemma 7.1, $\|\hat{G}\|_{\infty}=\alpha_{1} /\left(l \beta_{1}\right)$ (as may be recalled from eq 10 ). Therefore, we conclude that for the nonlinear one-step cascade, the $f^{2}$ gain is still $\|\hat{G}\|_{\infty}$. The generalization to a cascade of length $n$ may be found in ref 24 .

Acknowledgment. This work is partially supported by NIH Grant P20 GM64375 and Aventis. M. Chaves and E.D. Sontag were supported in part by the U.S. Air Force under Grant F49620-01-1-0063.

Supporting Information Available: Proof of the equality $\mathbf{C}_{\max }(l, R)=\mathbf{C}_{*}(l, R)$, properties of the function $f(k)$, minimization of $\sigma_{0}$, minimization of $F(n, M)$, and Laplace transforms and transfer functions. This material is available free of charge via the Internet at http://pubs.acs.org.

\section{References and Notes}

(1) Pouyssegur, P.; Lenormand, P. Fidelity and Spatio-Temporal Control in MAP Kinase (ERKs) Signaling. Eur. J. Biohem. 2003, 270, 3291-3299.

(2) Cohen, P. Protein Kinases - The Major Drug Targets of the TwentyFirst Century? Nat. Rev. Drug Discovery 2002, 1, 309-315.

(3) Foster, M. L.; Halley, F.; Souness, J. E. Potential of p38 Inhibitors in the Treatment of Rheumatoid Arthritis. Drug News Perspectives 2000 13 (8) 488-497.

(4) Ptashne, M.; Gann, A. Imposing Specificity on Kinases. Science 2003, 299, 1025-1027.

(5) Karandikar, M.; Cobb, M. H. Scaffolding and Protein Interactions in MAP Kinase Modules. Cell Calcium 1999, 26, 219-226.

(6) Asthagiri, A. R.; Lauffenburger, D. A. A Computational Study of Feedback Effects on Signal Dynamics in a Mitogen-Activated Protein Kinase (MAPK) pathway model. Biotechnol. Prog. 2001, 17 (2) 227-239.

(7) Bhalla, U. S.; Iyengar, R. Robustness of the Bistable Behavior of a Biological Signaling Feedback Loop. Chaos 2001, 11 (1), 221-226.

(8) Bhalla, U. S.; Ram, P. T.; Iyengar, R. MAP Kinase Phosphatase as a Locus of Flexibility in a Mitogen-Activated Protein Kinase Signaling Network. Science 2002, 297 (5583), 1018-1023.

(9) Bruggeman, F. J.; Westerhoff, H. V.; Hoek, J. B.; Kholodenko, B. N. Modular Response Analysis of Cellular Regulatory Networks. J. Theor. Biol. 2002, 218 (4) 507-520.

(10) Heinrich, R.; Neel, B. G.; Rapoport, T. A. Mathematical Models of Protein Kinase Signal Transduction. Mol. Cell 2002, 9, 957-970.

(11) Huang, C-Y. F.; Ferrell, J. E., Jr. Untrasensitivity in the MitogenActivated Protein Kinase Cascade. Proc. Natl. Acad. Sci. U. S. A. 1996 93, 10078-10083.

(12) Kholodenko, B. N. Four-Dimensional Organization of Protein Kinase Signaling Cascades: The Roles of Diffusion, Endocytosis and Molecular Motors. Exp. Biol. J. 2003, 206 (12) 2073-2082.

(13) Kholodenko, B. N.; Hoek, J. B.; Westerhoff, H. V.; Brown, G. C. Quantification of Information Transfer via Cellular Signal Transduction Pathways. FEBS Lett. 1997, 414 (2), 430-434.

(14) Levchenko, A.; Bruck, J.; Sternberg, P. W. Scaffold Proteins May Biphasically Affect the Levels of Mitogen-Activated Protein Kinase Signaling and Reduce Its Threshold Properties. Proc. Natl. Acad. Sci. U. S. A. 2000, 97, 5818-5823.

(15) Ingalls, B. P. Linear Input-Output Models of Biochemical Networks. J. Phys. Chem. 2004, 108, 1143-1152.

(16) Ingalls, B. P.; Sauro, H. S. Sensitivity Analysis of Stoichiometric Networks: An Extension of Metabolic Control Analysis to Nonequilibrium Trajectories. J. Theor. Biol. 2003, 222, 23-36.

(17) Anderson, B. D. O.; Moore, J. B. Linear Optimal Control; Prentice Hall: Englewood Cliffs, NJ, 1971.

(18) Sontag, E. D. Mathematical Control Theory: Deterministic Finite Dimensional Systems, 2nd ed.; Springer, NY, 1998.

(19) Doyle, J.; Francis, B.; Tannenbaum, A. Feedback Control Systems, MacMillan Publishing Co, 1992. Also available on http://www.control.utoronto.ca/people/profs/francis/dft.html

(20) Khalil, H. Nonlinear Systems, 2nd ed.; Prentice-Hall: Upper Saddle River, NJ, 1996.

(21) Angeli, D.; Sontag, E. D. Monotone Control Systems. IEEE Trans. Autom. Control 2003, 48, 1684-1698.

(22) van der Schaft, A $L_{2}$-Gain and Passivity Techniques in Nonlinear Control, (Lecture Notes in Control and Information Sciences, 218); SpringerVerlag: London, 1996.

(23) Sontag, E. D. Stability and Stabilization: Discontinuities and the Effect of Disturbances, in Nonlinear Analysis, Differential Equations, and Control; Clarke, F. H., Stern, R. J., Eds.; Kluwer: Dordrecht, 1999; pp $551-598$.

(24) Sontag, E. D.; Chaves, M. Computation of Amplification for Systems Arising from Cellular Signaling Pathways. Submitted for publication. 